\title{
In-Process Diameter Measurement Technique for Micro-Optical Fiber with Standing Wave Illumination
}

\author{
Masaki Michihata $^{1}$ (D) Zhao Zheng $^{1} \cdot$ Daiki Funaiwa $^{1} \cdot$ Sojiro Murakami ${ }^{1} \cdot$ Shotaro Kadoya $^{2} \cdot$ Satoru Takahashi $^{2}$
}

Received: 18 September 2020 / Revised: 12 October 2020 / Accepted: 23 October 2020 / Published online: 13 January 2021

(c) The Author(s) 2021, corrected publication 2022

\begin{abstract}
In this paper, we propose an in-process measurement method of the diameter of micro-optical fiber such as a tapered optical fiber. The proposed technique is based on analyzing optically scattered light generated by standing wave illumination. The proposed method is significant in that it requires an only limited measurement range and does not require a high dynamic range sensor. These properties are suitable for in-process measurement. This experiment verified that the proposed method could measure a fiber diameter as stable as $\pm 0.01 \mu \mathrm{m}$ under an air turbulence environment. As a result of comparing the measured diameter distribution with those by scanning electron microscopy, it was confirmed that the proposed method has a measurement accuracy better than several hundred nanometers.
\end{abstract}

Keywords Micro-fiber $\cdot$ In-process measurement $\cdot$ Mi scattering theory $\cdot$ Standing wave $\cdot$ Diameter

\section{Introduction}

A tapered optical fiber is a fiber having a partially thinned part such as shown in Fig. 1b [1]. The diameter of the thinned part is commonly smaller than a few micrometers. In the principle of the optical fiber, there is a core and a clad. Light propagates in the core with a total reflection at a boundary to the clad. In the thinned part, the fiber itself serves as a core, and surrounding air acts as a clad, where the evanescent light is generated outside of the optical fiber. Using this property, a tapered optical fiber is used for nano/ micro tweezers [2], plasmonic device [3], nano-cavity [4], optical coupler [5], sensor [6], and so on. A tapered optical fiber has also attracted researchers because the energy density can be increased inside the fiber so that non-linear optical phenomenon will occur easily. This property is applied

Electronic supplementary material The online version of this article (https://doi.org/10.1007/s41871-020-00081-4) contains supplementary material, which is available to authorized users.

Masaki Michihata

michihata@ nanolab.t.u-tokyo.ac.jp

1 Department of Precision Engineering, The University of Tokyo, 7-3-1 Hongo, Bunkyo, Tokyo 113-8654, Japan

2 Research Center for Advanced Science and Technology, The University of Tokyo, 4-6-1 Komaba, Meguro, Tokyo 153-8904, Japan for supercontinuum light generation [7], laser cavity [8], and so on.

A tapered optical fiber is typically fabricated by means of heating and pulling methods. A normal optical bare fiber (Fig. 1a) is heated with either a frame or a ceramic heater, and a $\mathrm{CO}_{2}$ laser for melting [1]. Then, the optical fiber is axially stretched. A feature of a tapered optical fiber is simply a cylinder or tapered cylinder, which means that diameter distribution is the most important as well as material properties in order to assure the function of a tapered optical fiber. To control the diameter distribution, the fine adjustment of controlling parameters in the fabrication process is essential [9], that is, in-process measurement of diameter for feedback control is required for accurate manufacturing. Regarding in-process measurement, since the diameter of a tapered optical fiber is in the range of $100 \mathrm{~nm}$ to several micrometers [1], a conventional laser micrometer is no longer used. Hence, many methods have been proposed so far. Diameter measurement using scanning electron microscopy (SEM) is the most typical choice because of versatility, reliability, and high resolution [10, 11], however, it is not applicable to in-process measurement. Most of the measurement techniques are based on optical methods, which are classified into two categories. One is based on Mie scattering analysis and the other is based on propagating light analysis. As is well known, Mie scattering ties the light scattering pattern with a diameter 


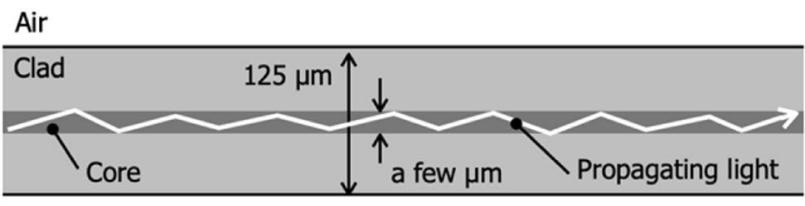

(a) Single mode fiber

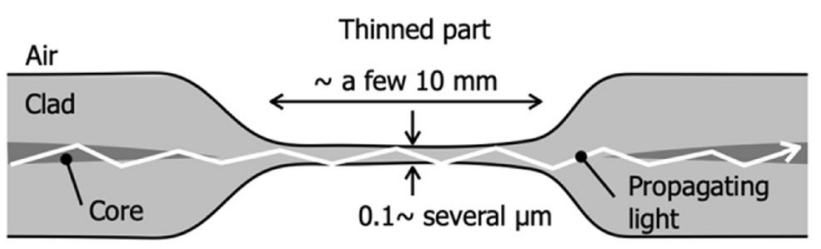

(b) Tapered optical fiber

Fig. 1 Structure of tapered optical fiber compared with single-mode fiber

of a fiber rigorously [12]. Many methods based on forward and back-scattered light analyses have been proposed [13-21]. Warken and Giessen achieved a measurement accuracy of $50 \mathrm{~nm}$ using forward-scattering light analysis [20]. Azzoune et al. achieved a measurement accuracy of $50 \mathrm{~nm}$ using forward-scattering light from white light illumination [21]. Mie scattering analysis is quite useful for the measurement of diameter. The scattering patterns, however, are so complex that it is necessary to measure the wide angular range of the scattering pattern. Therefore, we explore another method that is more suitable for in-process measurement. On the other hand, as another approach, there is propagating light analysis. Wiedemann et al. characterized the diameter using the generated higher-harmonic waves [11] and Vahara et al. proposed a strong measurement method using optical backscatter reflectometry [22]. These methods are well developed; however, in some drawbacks, for example, the material limitation and measurement speed prevent application into the in-process measurement. In addition, propagating light analysis tends to use expensive measurement instruments. Other interesting methods are those using whispering gallery mode [23] and external cavity [24], although these are difficult to install into in-process measurement because a fiber or a cavity must be very close to the measured fiber.

In this paper, we propose a novel method suited to in-process measurement based on the analysis of Mie-scattering light intensity distribution generated by standing wave illumination. The aim of this paper is to show the applicability of the proposed method for a micro-optical fiber. In the following chapters, the scattered light patterns by the standing wave and single beam illumination were compared to highlight the properties of the proposed method and experiments were conducted to show the feasibility and performance of the proposed method.

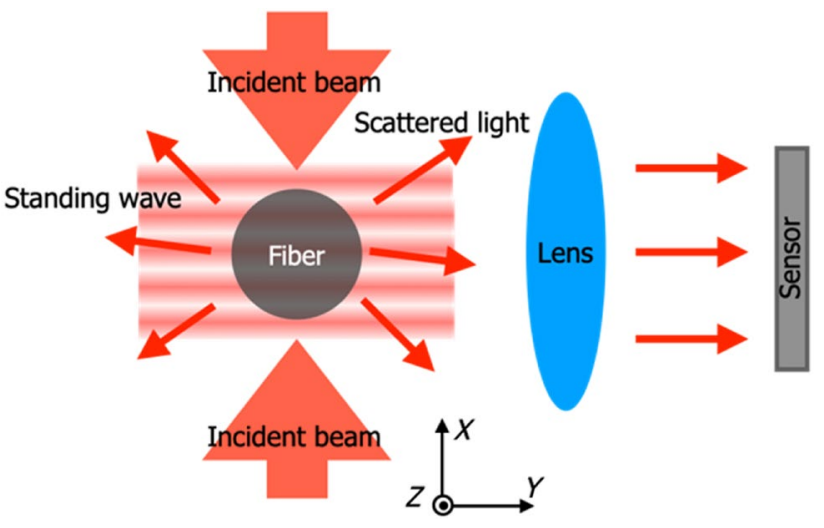

Fig. 2 Concept of the proposed measurement method for micro-fiber diameter

\section{Proposed Measurement Concept for the Diameter of Micro-Fiber}

As explained, the diameter distribution of a tapered optical fiber is desired for in-process measurement. For this, high measuring speed and a wide working distance are required. Therefore, there should be no mechanical scanning mechanism. The narrower the measuring angular range, the better. A measurement method on the basis of Mie-scattering light analysis is expected to be of high precision. However, for accurate measurement of a fiber diameter using Miescattering, a mechanism of scanning the light detector is often used to measure over a wide angular range, and careful calibration of the measured angle is also important. These requirements are not suitable for in-process measurement. Therefore, we proposed a novel measuring method for micro/nano-optical fiber using Mie-scattering light intensity generated by standing wave illumination. Commonly, a single plane wave is illuminated to the fiber. Instead, in this study, the counter-propagating beams are illuminated, as shown in Fig. 2, which is also recognized as a standing wave illumination. Then the light scattered along the $Y$-axis is measured. Because of the standing wave illumination, the scattered light intensity pattern tends to have periodicity, which makes the analysis of the scattering pattern simpler. The scattered light is measured from a direction orthogonal to the direction of the standing wave. The specific information to estimate a fiber diameter can be retrieved from scattered light intensity distribution within a certain angular range centered on $Y$-axis, so no mechanical scanning is required to measure the scattering light distribution. The detail of these is analyzed in the next chapter. 


\section{Theoretical Analysis of Scattering Light from Micro-Fiber}

\subsection{Theoretical Equation of Mie-Scattering Light Intensity}

The theoretical analysis based on Mie-scattering theory is described here. An optical fiber is approximated to a homogeneous infinite length dielectric cylinder and aligned with the $Z$-axis (see Fig. 2). Laser beams are illuminated from both the positive and negative direction of the $X$-axis to generate a standing wave. When considering a single beam, the illumination is incident on the fiber from the positive to negative direction of the $X$-axis. The laser beams are a polarized plane wave. From the scattering light field, the far-field distribution of the scattered light by the fiber in the $X Y$ plane was calculated. The theoretical scattered light intensity by a single plane wave and by the standing wave was shown.

The far-field scattered light intensity distribution with a single plane wave, $I_{p w}$, is calculated based on the Mie-scattering theory using the following equation $[12,25]$.

$$
\begin{aligned}
& I_{p w}=\frac{\lambda_{0}}{n \pi^{2} r}\left|\sum_{m=-\infty}^{\infty} a_{m} e^{i m \theta}\right|^{2} I_{i n c} \\
& a_{m}= \begin{cases}\frac{n J_{m}\left(k_{2} R\right) J_{m}^{\prime}\left(k_{1} R\right)-J_{m}\left(k_{1} R\right) J_{m}^{\prime}\left(k_{2} R\right)}{n J_{m}^{\prime}\left(k_{2} R\right) H_{m}^{(1)}\left(k_{1} R\right)-J_{m}\left(k_{2} R\right) H_{m}^{(1)}\left(k_{1} R\right)} & \cdots \vec{E} \| Z, \vec{H} \perp Z \\
\frac{J_{m}\left(k_{1} R\right) J_{m}^{\prime}\left(k_{2} R\right)-n J_{m}\left(k_{2} R\right) J_{m}^{\prime}\left(k_{1} R\right)}{J_{m}^{\prime}\left(k_{2} R\right) H_{m}^{(1)}\left(k_{1} R\right)-n J_{m}\left(k_{2} R\right) H_{m}^{(1)^{\prime}}\left(k_{1} R\right)} & \cdots \vec{E} \perp Z, \vec{H} \| Z\end{cases}
\end{aligned}
$$

where, $\lambda_{0}$ is the wavelength in vacuum, $k$ is the wavenumber, and subscript 1 or 2 indicates inside or outside of the fiber, respectively, $R$ is the radius of the fiber, $\theta$ is the scattering angle, $n$ is the refractive index of the fiber, $I_{i n c}$ is the light intensity of the incident laser, $J_{m}$ is the Bessel of order $m$, prime denotes the derivative of the function with respect to its argument, and $H_{m}^{(1)}$ is the Hankel function of first kind of order $m$.

Far-field scattered light intensity by the standing wave, $I_{s w^{*}}$. can be calculated using Eq. (3) and (4).

$$
\begin{aligned}
& I_{s w}=\frac{\lambda_{0}}{n \pi^{2} r}\left|\sum_{m=-\infty}^{\infty}\left[e^{i \phi_{1}}(-1)^{m}+e^{i \phi_{2}}\right] b_{m} e^{i m \theta}\right|^{2} I_{i n c} \\
& b_{m}= \begin{cases}\frac{J_{m}\left(k_{1} R\right) J_{m}^{\prime}\left(k_{2} R\right)-\alpha J_{m}\left(k_{2} R\right) J_{m}^{\prime}\left(k_{1} R\right)}{\alpha J_{m}^{\prime}\left(k_{1} R\right) H_{m}^{(2)}\left(k_{2} R\right)-J_{m}\left(k_{1} R\right) H_{m}^{(2)}\left(k_{2} R\right)} & \cdots \vec{E} \| Z, \vec{H} \perp Z \\
\frac{\alpha J_{m}\left(k_{1} R J_{m}^{\prime}\left(k_{2} R\right)-J_{m}\left(k_{2} R\right) J_{m}^{\prime}\left(k_{2} R\right)\right.}{J_{m}^{\prime}\left(k_{1} R\right) H_{m}^{(2)}\left(k_{2} R\right)-\alpha J_{m}\left(k_{1} R\right) H_{m}^{(2)}\left(k_{2} R\right)} & \cdots \vec{E} \perp Z, \vec{H} \| Z\end{cases}
\end{aligned}
$$

where, $\varphi_{1}$ and $\varphi_{2}$ are the phases of two incident beams. $H_{m}^{(2)}$ is the Hankel function of second kind of order $m . \alpha$ is $\left(\varepsilon_{1} \mu_{2} / \varepsilon_{2} \mu_{1}\right)^{1 / 2} \cdot \varepsilon$ and $\mu$ are permittivity and permeability, respectively. The phase of the standing wave, which is generated by the two counter-propagating incident beams, can be determined by the relative phase difference of $\varphi_{1}$ and $\varphi_{2}$. In this paper, the phase $\varphi_{1}$ was always set to zero, and the phase $\varphi_{2}$ was changed to differentiate the phase of the standing wave illumination.

\subsection{Scattered light intensity distribution}

For exploiting the measurement principle, the scattered light intensity distributions with different diameters of optical fiber were calculated using Eqs. (1)-(4) for diameters ranging from 0.5 to $7 \mu \mathrm{m}$. For the laser source, an $\mathrm{He}-\mathrm{Ne}$ laser was used and the wavelength of the laser was set to $632.8 \mathrm{~nm}$. The polarization of the incident beams was parallel to the $Z$-axis. The refractive index of optical fiber was set to 1.454 . Figure 3 shows the calculated angular distribution of scattering light intensity. The red and black solid lines indicate the scattered light intensity pattern by the standing wave illumination and single plane wave, respectively. The single plane wave was incident from a direction of $0^{\circ}$, and the laser beams were incident from a direction of $0^{\circ}$ and $180^{\circ}$ to generate the standing wave. Scattered light intensity was displayed logarithmically. Regarding the plane wave illumination, the scattered light intensity patterns are simply that there are several peaks and valleys for small diameters, however, it becomes more complex for diameters larger than $0.8 \mu \mathrm{m}$, where it seems difficult to obtain the characteristic of the scattered light intensity pattern from just positions of the peaks and valleys. Therefore, it implied requiring the high dynamic range measurement of the scattering light intensity and wide measuring range for a diameter measurement based on Mie-scattering light pattern by singleplane wave illumination. On the other hand, the scattered light intensity patterns by standing wave illumination are simpler than the one by plane wave illumination, basically consisting of peaks and valleys. It is also noted that peaks and valleys appeared particularly concentrated around $90^{\circ}$. The reason for the most characteristic pattern that appeared in the direction perpendicular to the axis of the standing wave is because the peaks and valleys are caused not only by the Mie-scattering pattern but also by the light intensity of the standing wave illumination. Therefore the most characteristic pattern appeared in the direction of perpendicular to the axis of the standing wave.

Figure 4 shows the calculated angular distribution of scattered light intensity with different phases of the standing wave illumination, practically the phase $\varphi_{2}$ in Eq. (3) was changed. As shown, the scattered light intensity pattern was changing with varying the phase of the standing wave. The position of peaks and valleys is shifted gradually, but still, these exist around $90^{\circ}$. Accordingly, it is expected to 


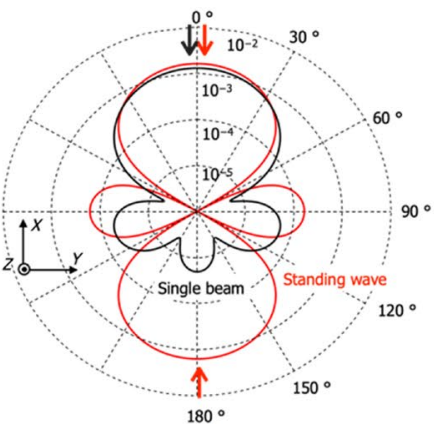

(a) $0.5 \mu \mathrm{m}$

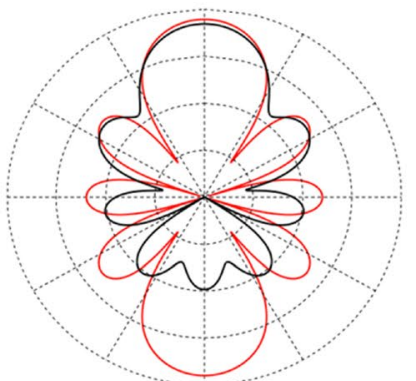

(e) $0.9 \mu \mathrm{m}$

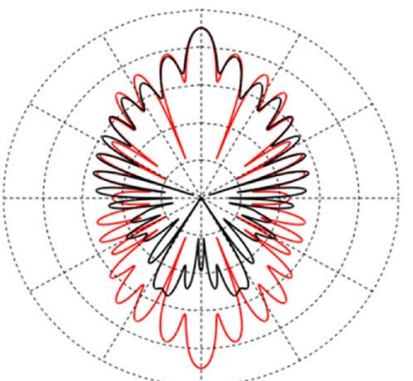

(i) $4.0 \mu \mathrm{m}$

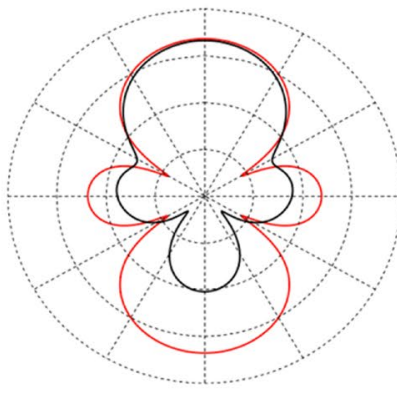

(b) $0.6 \mu \mathrm{m}$

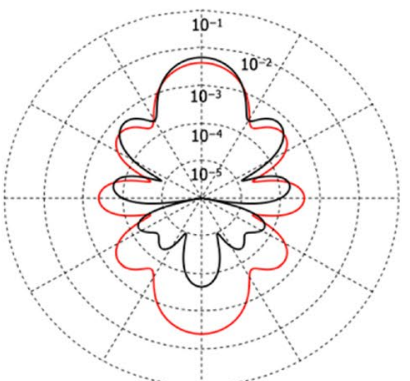

(f) $1.0 \mu \mathrm{m}$

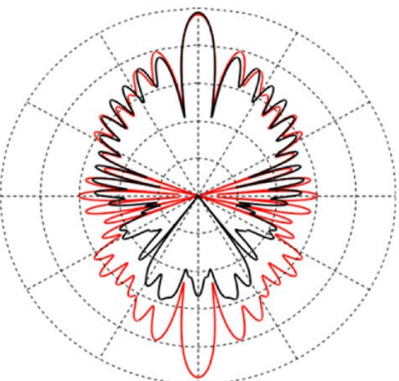

(j) $5.0 \mu \mathrm{m}$

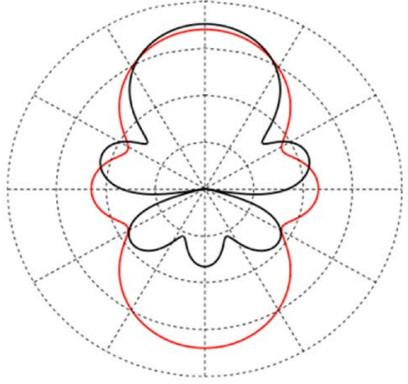

(c) $0.7 \mu \mathrm{m}$

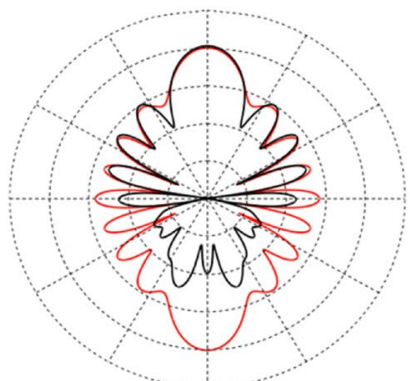

(g) $2.0 \mu \mathrm{m}$

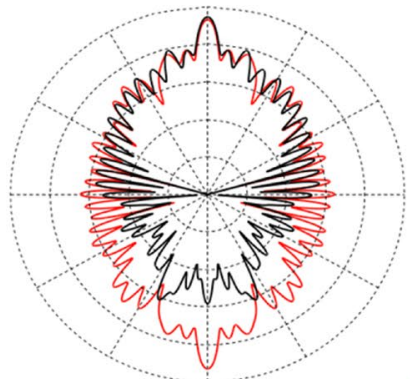

(k) $6.0 \mu \mathrm{m}$

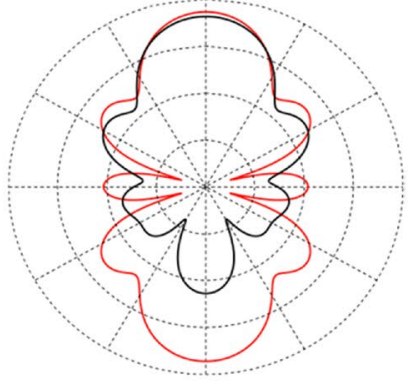

(d) $0.8 \mu \mathrm{m}$

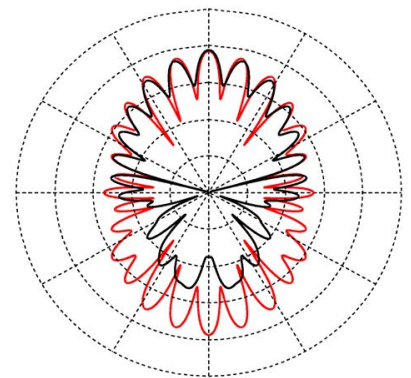

(h) $3.0 \mu \mathrm{m}$

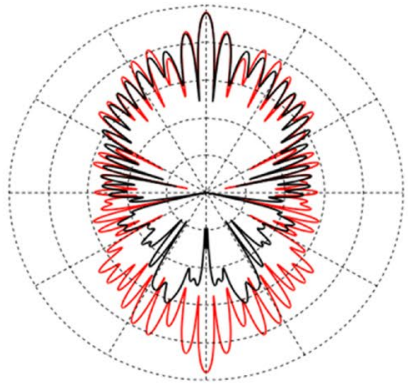

(1) $7.0 \mu \mathrm{m}$

$\rightarrow$ Direction of incident beam of standing wave

$\rightarrow$ Direction of incident beam of single plane wave

Fig. 3 Angular distribution of scattered light intensity caused by standing-wave and single plane-wave illumination

determine the diameter of fiber via the observation of the scattered light from $60^{\circ}$ to $120^{\circ}$ centered on $90^{\circ}$, which can be realized by using an objective lens of numerical aperture (NA) 0.55 .

Figure 5 shows the scattered light intensity pattern around $90^{\circ}$ at the far-field sensor plane. The horizontal and vertical axes are the scattered angle and the light intensity, respectively. Here, the light intensity was shown on a linear scale. Simulated conditions are the same as in Fig. 3. As seen, the scattered light intensity pattern is symmetric in the standing wave, but asymmetric in the single beam, which means that light intensity is on the same level over the measured range. It is an effective property for in-process measurement because it is possible to use the one sensor array such as a CMOS sensor without troublesome tuning of sensitivity. These features are the same for different phase conditions of the standing wave illumination. In the supplemental figure, the scattered light distribution in a case of $7 \mu \mathrm{m}$ in diameter with different phase $\varphi_{2}$ is shown. The scattered light intensity was on the same level and the distribution was composed of peaks and valleys.

\subsection{Principle of Measurement of Fiber Diameter}

In terms of fiber diameter estimation based on the analysis of the intensity distribution, as previously reported [26], by measuring the averaged pitch of the scattered light pattern, the diameter of a fiber can be roughly estimated within an accuracy of a few micrometers. In this paper, it was addressed to develop a more accurate method. As described earlier, the scattered light intensity pattern by the standing wave can be characterized by peaks and valleys. Therefore, 


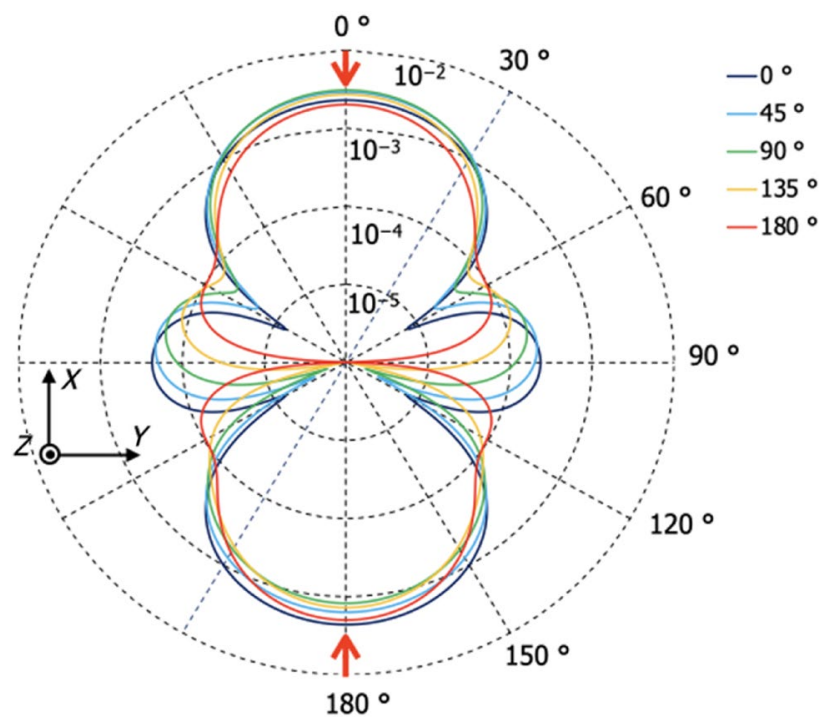

Fig. 4 Angular distribution of scattered light intensity caused by standing waves with different phases in a case of a fiber diameter of $0.6 \mu \mathrm{m}$. The legend indicates the phase value of one incident beam, $\varphi_{2}$

the measurement method using an angular position of peaks and valleys was considered. Firstly, the theoretical positions of these were calculated as shown in Fig. 6. Even for relatively thinner (Fig. 6a) and thicker (Fig. 6b) diameter of the fiber, the peak and valley vary with changing diameters and its changes are complex, therefore we decided to use the fitting method. Prior to the measurement, the theoretical positions of peaks or valleys were calculated with different fiber diameters and different phases of standing wave illumination to make a reference table. The measured angular positions of peaks and valleys would be compared with those on the reference table, and then the root mean square (RMS) error was calculated. The phase of the standing wave and fiber diameter that make the RMS error the smallest are explored. Particularly, the angular distances between neighboring peaks or valleys were used instead of the absolute angular positions of peaks or valleys because there may be certain alignment errors of the optical system. In this paper, using the valley of the scattered light intensity pattern, the feasibility of this measurement method was confirmed.

\section{Experimental Setup}

To verify the feasibility of the proposed method, the scattered light intensity pattern was experimentally measured. Figure 7 shows the experimental setup. For the light source, an $\mathrm{He}-\mathrm{Ne}$ laser was used. The output power was approximately $1 \mathrm{~mW}$ and the wavelength was $632.8 \mathrm{~nm}$. After polarization and beam diameter adjustment, the beam was split into two beams. The diameter of the beam was approximately $1 \mathrm{~mm}$. The two beams were aligned to co-axially incident on the fiber from opposite directions. The polarization of the beams was adjusted parallel to the axis of the optical fiber. The scattered light from the optical fiber was collected by the objective lens. In this paper, the NA of the objective lens used was 0.55 so that the objective lens could collect the scattered light over $\pm 33.3^{\circ}$ in angle. The working distance of the objective lens was $13 \mathrm{~mm}$. CMOS 1 was set at the conjugate plane of the Fourier plane of the objective lens in order to measure the scattered light intensity pattern. CMOS 2 was set to image the optical fiber with a magnification of $\times 100$.

Figure 8 shows the optical image of the tapered optical fiber (Fig. 8b) and the distribution of scattered light (Fig. 8a) taken with the developed system. In Fig. 8b, the edges of the fiber were red because of the strong scattering of the incident beams from both sides. In Fig. 8a, the scattered light intensity pattern taken with CMOS 1 was clearly observed. This intensity distribution on the sensor plane was converted into scattered angle distribution based on the Abbe's sine condition and the magnification of the optical system.

\section{Experiment}

\subsection{Measurement property of scattered light intensity distribution}

First, the measurement of the scattered light intensity and the performance of fitting with the reference table were examined. The reference table was prepared with a diameter step of $0.01 \mu \mathrm{m}$ and a phase step of $10^{\circ}$. Second, the scattered light intensity pattern was measured. As a measurement sample, the self-made tapered optical fiber whose smallest diameter was approximately $5 \mu \mathrm{m}$ was used. The measured intensity distribution is shown in Fig. 9, from which the angular positions of valleys were obtained. Experimentally obtained angular distances of the valleys were compared with those on the reference table to find the most matched fiber diameter and the phase of the standing wave. Based on the measured intensity shown in Fig. 9, the fiber diameter was estimated to be $6.81 \mu \mathrm{m}$. The phase of the standing wave was estimated as $\varphi_{2}=150^{\circ}$. In this case, the RMS error was $0.19^{\circ}$. Throughout all measurements, RMS errors were less than $0.2^{\circ}$. The theoretical curves using obtained parameters are also shown in Fig. 9. It can be confirmed that the measured intensity distribution was well fitted with the theoretically estimated intensity distribution in terms of the position of the valleys. However, the shape of the intensity distribution did not agree well. The reasons for this could be, for example, non-uniform standing wave illumination due to the difference of laser power of two incident beams 


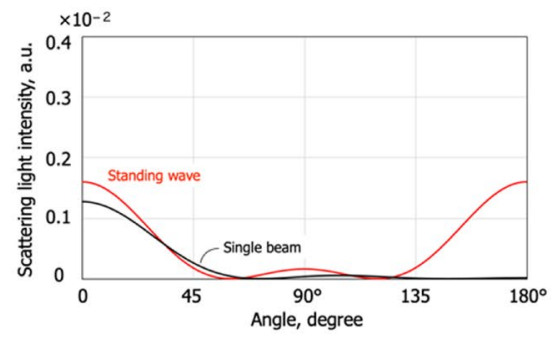

(a) $0.5 \mu \mathrm{m}$

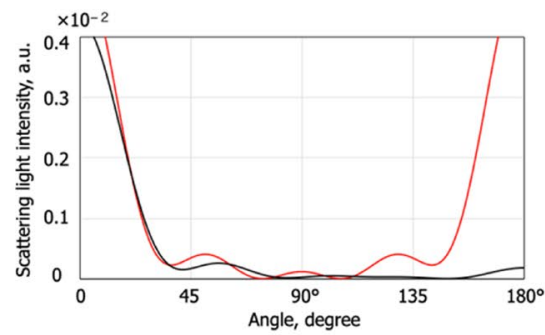

(d) $0.8 \mu \mathrm{m}$

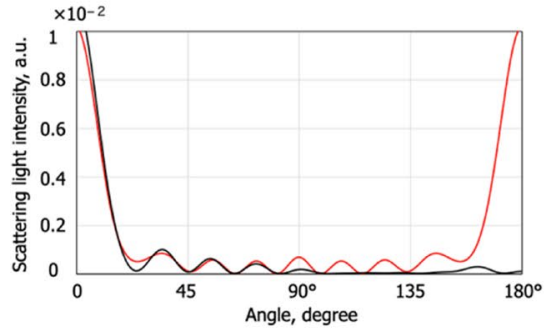

(g) $2.0 \mu \mathrm{m}$

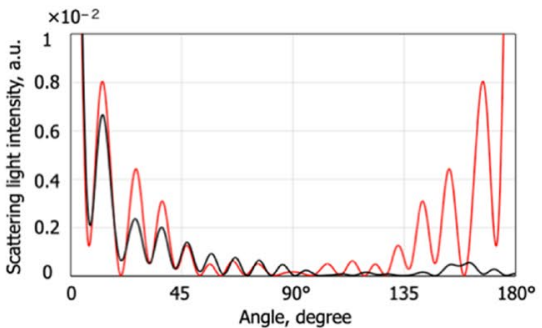

(j) $5.0 \mu \mathrm{m}$

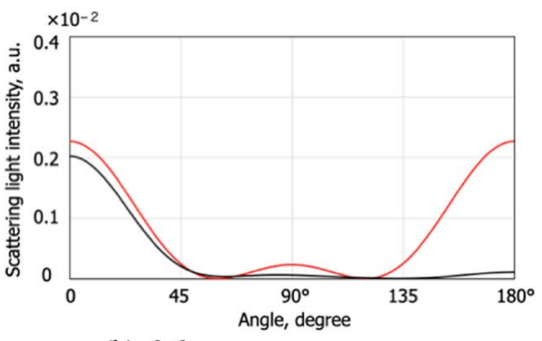

(b) $0.6 \mu \mathrm{m}$

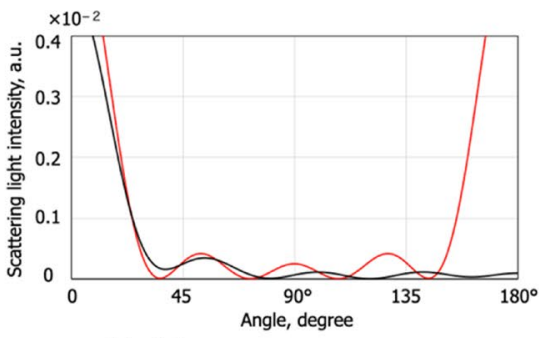

(e) $0.9 \mu \mathrm{m}$

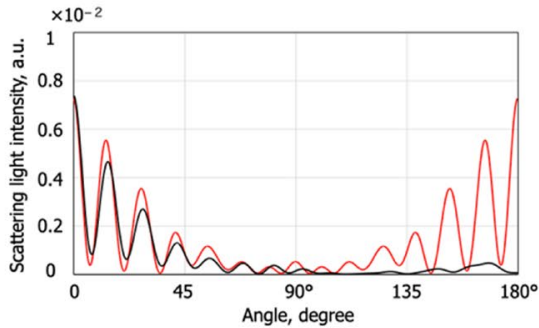

(h) $3.0 \mu \mathrm{m}$

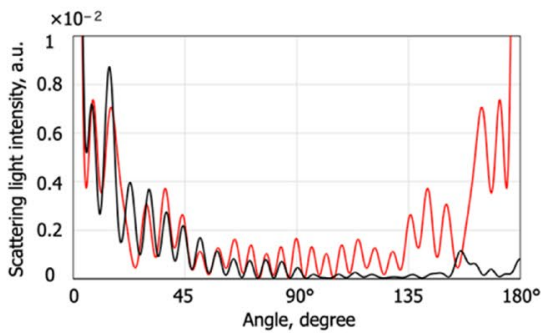

(k) $6.0 \mu \mathrm{m}$

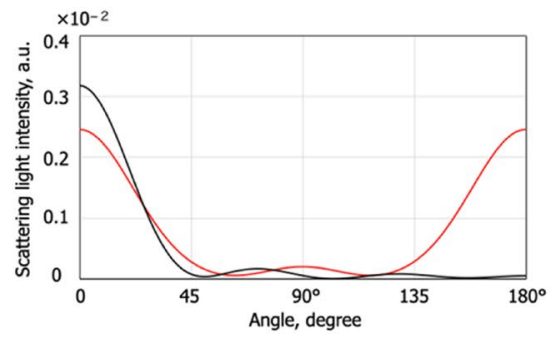

(c) $0.7 \mu \mathrm{m}$

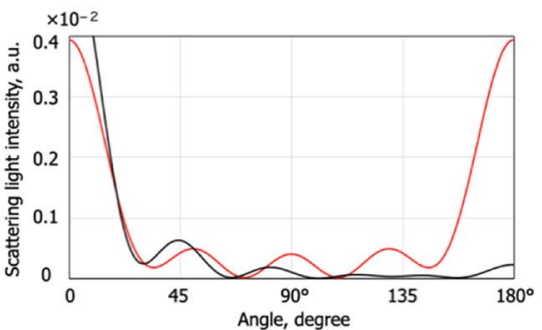

(f) $1.0 \mu \mathrm{m}$

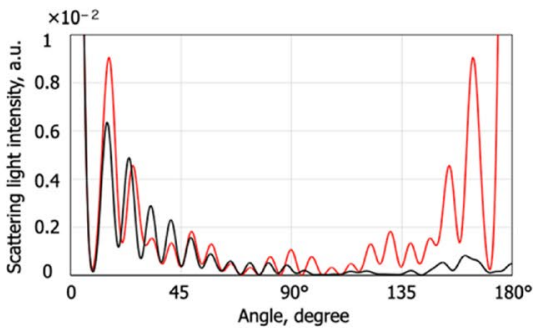

(i) $4.0 \mu \mathrm{m}$

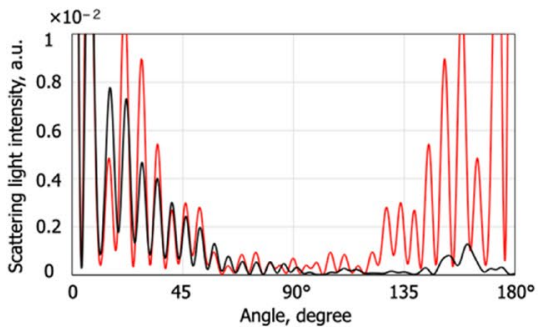

(1) $7.0 \mu \mathrm{m}$

Fig. 5 Scattered light intensity distribution caused by standing-wave and single plane-wave illumination on sensor plane. Red and black solid lines indicate the ones by standing-wave and single plane-wave illumination, respectively

or misalignment of the optical measurement system. Thus, it is hard to measure the intensity consistent with the theoretical intensities, and in this respect, it is not easy to measure the diameter in-process by measuring the scattered intensity distribution of Mie scattering by a single beam.

The proposed method uses standing wave illumination. The two incident beams interfered to form the standing wave, so disturbance of the surrounding air might affect the phase of the standing wave, resulting in fluctuation of the scattered light pattern. Figure 10 shows an example of the fluctuated scattered light intensity patterns. The measured position of the fiber is the same, but the patterns were different due to air turbulence. Table 1 shows the fitting result of the five images. As shown, the error of the estimated diameter was $\pm 0.01 \mu \mathrm{m}$. RMS errors were less than $0.18^{\circ}$, which assure that it is well fitted. Thus, even though there was the surrounding air disturbance, the measurement was performed stably. This property is essential to in-process measurement.

\subsection{Measurement of Diameter Distribution of Micro-Optical Fiber}

Finally, the diameter distribution of the tapered optical fiber was measured. The schematic of the measured sample is shown in Fig. 11. The tapered optical fiber was attached 


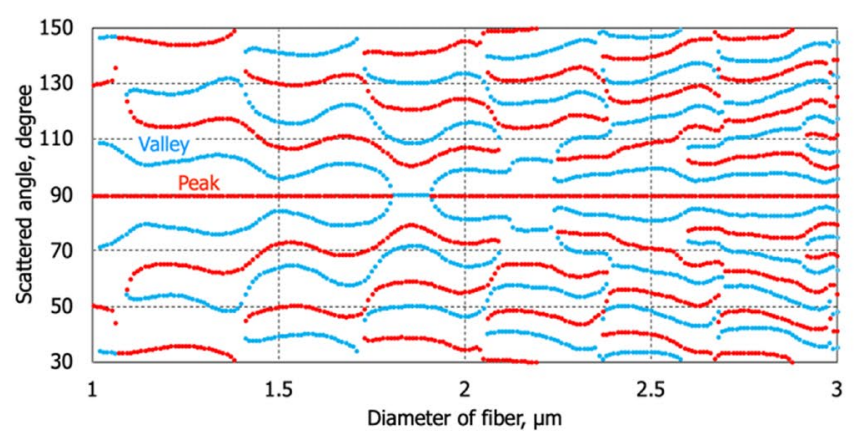

(a) From 1 to $3 \mu \mathrm{m}$.

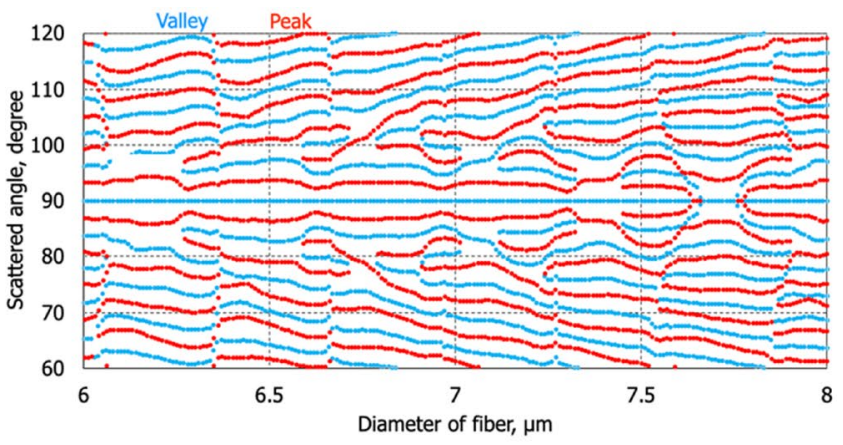

(b) From 6 to $8 \mu \mathrm{m}$.

Fig. 6 Calculated angular position of peaks and valleys of the scattered light intensity caused by standing wave illumination in the case of $\varphi_{2}=0$. The red and blue dots indicate the peak and valley, respectively

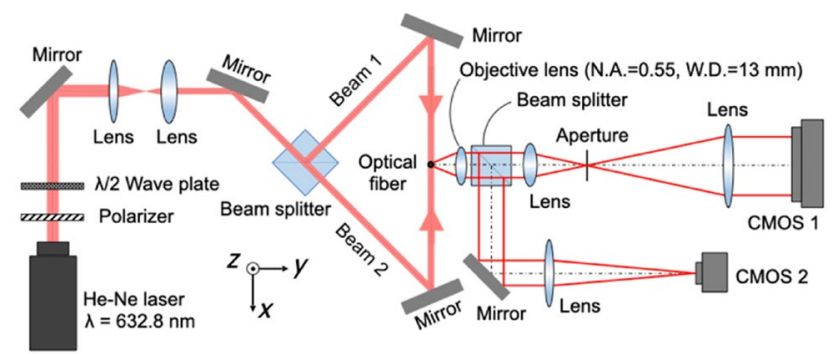

Fig. 7 Illustration of experimental setup

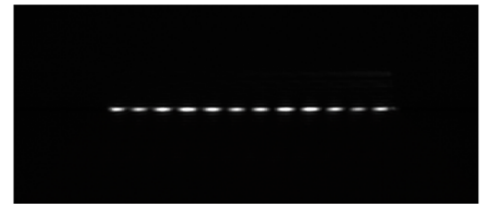

(a) Scattered light intensity taken by CMOS 1

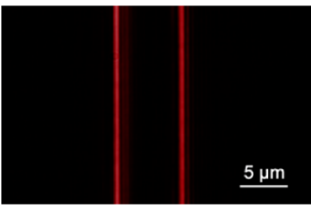

(b) Optical image of fiber taken by CMOS 2
Fig. 8 Experimentally obtained images by CMOS 1 and 2

to the holder that has the notch. At the notch area, the thinned part of the fiber was placed, so the measurable axial range was about $9 \mathrm{~mm}$. The measurement was carried out over the range of $7 \mathrm{~mm}$ in that area. The photo of the measurement is shown in Fig. 12. Fifteen points on the fiber were measured in total. The shift of the measured point was conducted by changing the fiber position using the micrometer stage. The same sample was also measured by SEM for comparison. The measurement results are shown in Fig. 13. Both measurement results agreed within several hundred nanometers. However, at $1 \mathrm{~mm}$ of the fiber position, the measurement result did not agree. The reason for this could not be clearly confirmed, but it was possible to infer that there was contamination or a scratch

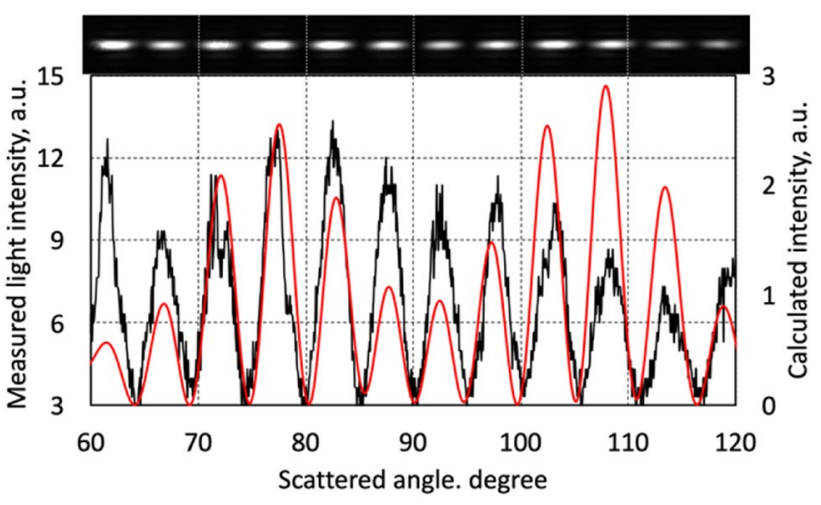

Fig. 9 Measured scattered light intensity distribution (black line) together with the theoretically calculated light intensity (red line) whose parameters were obtained by the fitting procedure

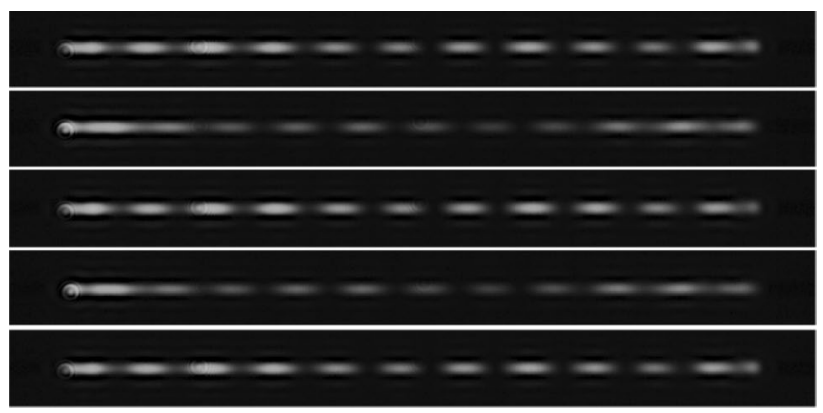

Fig. 10 The measured scattered light intensity patterns under air turbulence

on the fiber. As a result of the measurement, the feasibility of the proposed method was confirmed. Accuracy of the proposed measurement method was confirmed to be several hundred nanometers, which might be improved by increasing the signal-to-noise ratio of scattered light measurement and by modifying the optical system. To 
Table 1 Estimated parameters by fitting procedure

\begin{tabular}{llll}
\hline No. & $\begin{array}{l}\text { Estimated diameter } \\
(\mu \mathrm{m})\end{array}$ & Phase $\left(^{\circ}\right)$ & RMS error $\left(^{\circ}\right)$ \\
\hline 1 & 7.37 & 100 & 0.18 \\
2 & 7.36 & 130 & 0.16 \\
3 & 7.38 & 150 & 0.15 \\
4 & 7.37 & 150 & 0.17 \\
5 & 7.38 & 180 & 0.15 \\
\hline
\end{tabular}

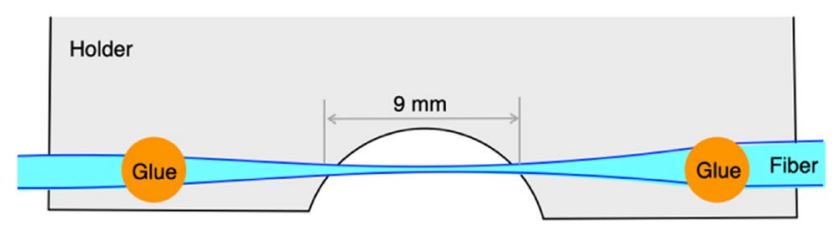

Fig. 11 Schematic of the measured tapered optical fiber

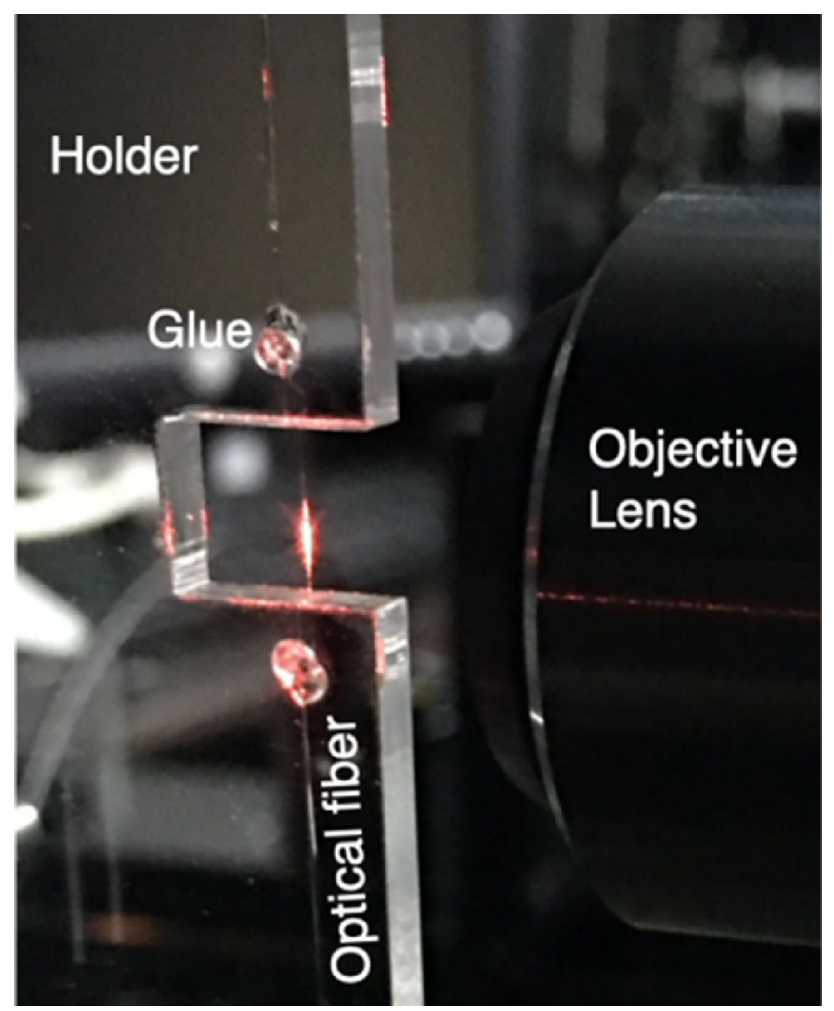

Fig. 12 Photo of the measured sample

transfer the scattered light intensity distribution into the sensor plane, the optical Fourier plane of the objective lens was observed. In this process, the scattered light from the different axial positions of the fiber was averaged. In the optical system of this study, approximately $30 \mu \mathrm{m}$ in the axial range were averaged. Considering the influence

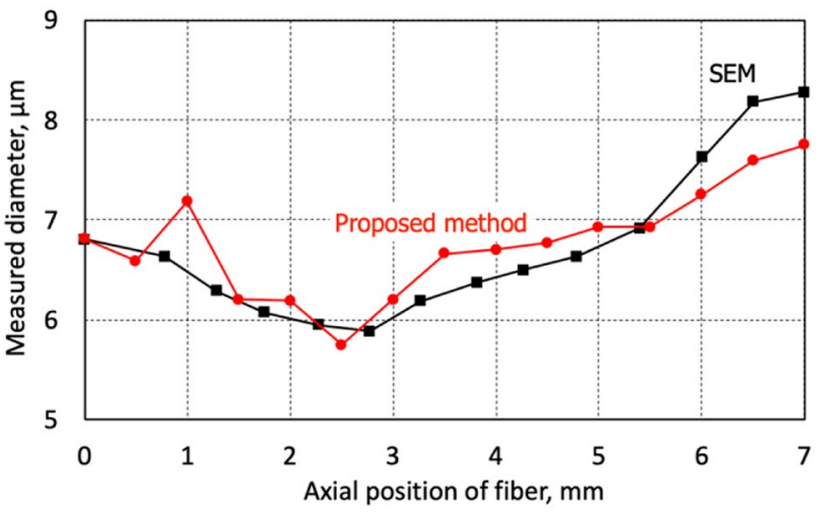

Fig. 13 Experimentally measured result of fiber diameter distribution. Red and black lines indicate the measured by the proposed method and by SEM, respectively

of fiber tilt or fiber diameter distribution, the measured scattered light intensity might be distorted or blurred. By solving this problem, the measurement accuracy would be possibly improved.

\section{Conclusions}

In this paper, we proposed a novel method suitable for in-process measurement based on the analysis of Mie-scattering light intensity distribution generated by standing wave illumination. The theoretical analysis revealed that the scattered light intensity pattern generated by the standing wave illumination was possible to be characterized using the peaks and valleys. The peaks and valleys appeared particularly concentrated around $90^{\circ}$ from the illumination direction, which implied that only a limited angular measurement range is necessary for the scattered light intensity. These properties are suitable for in-process measurement. In the experiment, the diameter estimation method based on the angular position of the valleys of the scattered light intensity distribution was examined. The measured angular positions of the valleys were compared with those on the reference table to find the fiber diameter and the phase of the standing wave illumination, which was successfully realized to find the diameter as stable as $\pm 0.01 \mu \mathrm{m}$ even under the air turbulence environment. Finally, the diameter distribution of the optical fiber was measured and compared with those by SEM, which confirmed that the proposed method has a measurement accuracy better than several hundred nanometers.

Acknowledgements This work was supported by A-STEP from JST and MEXT/JSPS KAKENHI (No. 18K18803, 20H02040). The authors thank Assistant Professor L. Jin from RCAST, the University of Tokyo, for providing the measured tapered optical fiber.

Conflict of interest Satoru Takahashi is an editorial board member for "Nanomanfucturing and Metrology" and was not involved in the edito- 
rial review, or the decision to publish this article. All authors declare that there are no competing interests.

Open Access This article is licensed under a Creative Commons Attribution 4.0 International License, which permits use, sharing, adaptation, distribution and reproduction in any medium or format, as long as you give appropriate credit to the original author(s) and the source, provide a link to the Creative Commons licence, and indicate if changes were made. The images or other third party material in this article are included in the article's Creative Commons licence, unless indicated otherwise in a credit line to the material. If material is not included in the article's Creative Commons licence and your intended use is not permitted by statutory regulation or exceeds the permitted use, you will need to obtain permission directly from the copyright holder. To view a copy of this licence, visit http://creativecommons.org/licenses/by/4.0/.

\section{References}

1. Wu X, Tong L (2013) Optical microfibers and nanofibers. Nanophotonics 2(5-6):407-428. https://doi.org/10.1515/nanop h-2013-0033

2. Frawley MC, Gusachenko I, Truong VG, Sergides M, Nic Chormaic S (2014) Selective particle trapping and optical binding in the evanescent field of an optical nanofiber. Opt Express 22(13):16322-16334. https://doi.org/10.1364/OE.22.016322

3. Li K, Zhou W, Zeng S (2018) Optical micro/nanofiber-based localized surface plasmon resonance biosensors: fiber diameter dependence. Sensors 18:3295. https://doi.org/10.3390/s18103295

4. Yalla R, Sadgrove M, Nayak KP, Hakuta K (2014) Cavity quantum electrodynamics on a nanofiber using a composite photonic crystal cavity. Phys Rev Lett 113:143601. https://doi.org/10.1103/ PhysRevLett.113.143601

5. Kobayashi Y, Michihata M, Zheng Z, Chu B, Takamasu K, Takahashi S (2019) Radial mode number identification on whispering gallery mode resonances for diameter measurement of microsphere. Meas Sci Technol 30(6):065201. https://doi. org/10.1088/1361-6501/ab1241

6. Chen GY, Ding M, Newson TP, Brambilla G (2013) A review of microfiber- and nanofiber-based optical sensors. Open Opt J 7(1):32-57. https://doi.org/10.2174/1874328501307010032

7. Birks TA, Wadsworth WJ, Russell PSJ (2000) Supercontinuum generation in tapered fibers. Opt Lett 25(19):1415-1417. https:// doi.org/10.1364/OL.25.001415

8. Harun SW, Lim KS, Jasim AA, Ahmad H (2010) Fabrication of tapered fiber-based ring resonator. Laser Phys 20:1629-1631. https://doi.org/10.1134/S1054660X10130050

9. Harun SW, Lim KS, Tio CK, Dimyati K, Ahmad H (2013) Theoretical analysis and fabrication of tapered fiber. Optik 124:538543. https://doi.org/10.1016/j.ijleo.2011.12.054

10. Girei SH, Shabaneh AA, Ngee-Lim H, Hamidon MN, Mahdi MA, Yaacob MH (2015) Tapered optical fiber coated with graphenebased nanomaterials for measurement of ethanol concentrations in water. Opt Rev 22:385-392. https://doi.org/10.1007/s1004 3-015-0075-8

11. Wiedemann U, Karapetyan K, Dan C, Pritzkau D, Alt W, Irsen S, Meschede D (2010) Measurement of submicrometre diameters of tapered optical fibres using harmonic generation. Opt Express 18(8):7693-7704. https://doi.org/10.1364/OE.18.007693

12. Frezza F, Mangini F, Tedeschi N (2018) Introduction to electromagnetic scattering: tutorial. J Opt Soc Am A 35(1):163-173. https://doi.org/10.1364/JOSAA.35.000163

13. Saekeang C, Chu PL (1979) Nondestructive determination of refractive index profile of an optical fiber: Backward light scattering method. Appl Opt 18(7):1110-1116. https://doi.org/10.1364/ AO.18.001110

14. Jasapara J, Monberg E, DiMarcello F, Nicholson JW (2003) Accurate noncontact optical fiber diameter measurement with spectral interferometry. Opt Lett 28(8):601-603. https://doi.org/10.1364/ OL.28.000601

15. Malek M, Coetmellec S, Allano D, Lebrun D (2003) Formulation of in-line holography process by a linear shift invariant system: application to the measurement of fiber diameter. Opt Commun 223:263-271. https://doi.org/10.1016/S0030-4018(03)01693-6

16. Butler DJ, Forbes GW (1998) Fiber-diameter measurement by occlusion of a Gaussian beam. Appl Opt 37(13):2598-2607. https ://doi.org/10.1364/AO.37.002598

17. Smithgall DH, Watkins LS, Frazee DE Jr (1977) High-speed noncontact fiber-diameter measurement using forward light scattering. Appl Opt 16(9):2395-2402. https://doi.org/10.1364/AO.16.00239 5

18. Swirniak G, Mroczka J (2016) Approximate solution for optical measurements of the diameter and refractive index of a small and transparent fiber. J Opt Soc Am A 33(4):667-676. https://doi. org/10.1364/JOSAA.33.000667

19. Świrniak G, Głomb G, Mroczka J (2014) Inverse analysis of light scattered at a small angle for characterization of a transparent dielectric fiber. Appl Opt 53(30):7103-7111. https://doi.org/10.1364/ AO.53.007103

20. Warken F, Giessen H (2004) Fast profile measurement of micrometer-sized tapered fibers with better than 50-nm accuracy. Opt Lett 29(15):1727-1729. https://doi.org/10.1364/OL.29.001727

21. Azzoune A, Delaye P, Pauliat G (2019) Optical microscopy for measuring tapered fibers beyond the diffraction limit. Opt Express 27(17):24403. https://doi.org/10.1364/OE.27.024403

22. Lai Y-H, Yang KY, Suh M-G, Vahala KJ (2017) Fiber taper characterization by optical backscattering reflectometry. Opt Express 25(19):22312-22327. https://doi.org/10.1364/OE.25.022312

23. Kavungal V, Farrell G, Wu Q, Mallik AK, Semenova Y (2018) Studies of geometrical profiling in fabricated tapered optical fibers using whispering gallery modes spectroscopy. Opt Fiber Technol 41:82-88. https://doi.org/10.1016/j.yofte.2018.01.007

24. Keloth J, Sadgrove M, Yalla R, Hakuta K (2015) Diameter measurement of optical nanofibers using a composite photonic crystal cavity. Opt Lett 40(17):4122-4125. https://doi.org/10.1364/ OL.40.004122

25. Uemura Y, Fujimura M, Hashimoto T, Kawai H (1977) Application of light scattering from dielectric cylinder based upon Mie and Rayleigh-Gans-Born theories to polymer systems: I: Scattering from a glass fiber. Polym J 10(3):341-351. https://doi. org/10.1295/polymj.10.341

26. Michihata M, Zheng Z, Takamasu K, Takahashi S (2019) In-process measurement technique of micro-fiber diameter with interfered scattering pattern of two beam irradiation. In: Proceedings of ISMTII: No.65 